\title{
Prevalence and Predictors of Sustained Smoking after a Cancer Diagnosis in Korean Men
}

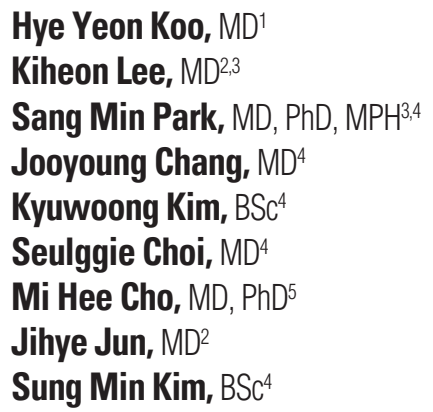

${ }^{1}$ Health Promotion Center, Gachon University Gil Medical Center, Incheon, ${ }^{2}$ Department of Family Medicine, Seoul National University Bundang Hospital, Seongnam, ${ }^{3}$ Department of Family Medicine, Seoul National University College of Medicine, Seoul, ${ }^{4}$ Department of Biomedical Sciences, Seoul National University Graduate School, Seoul, ${ }^{5}$ Kangbuk Samsung Hospital, Samsung CET Corporation Medical Clinic, Seoul, Korea

Correspondence: Kiheon Lee, MD Department of Family Medicine, Seoul National University Bundang Hospital, 82 Gumi-ro, 173beon-gil, Bundang-gu, Seongnam 13620, Korea Tel: 82-31-787-7801

Fax: 82-31-787-4834

E-mail: keyhoney@gmail.com

Received November 7, 2018

Accepted June 20, 2019

Published Online June 25, 2019

\begin{abstract}
Purpose
Although smoking has a significant impact on mortality and morbidity of cancer patients, many patients continue to smoke post-diagnosis. The purpose of this study was to investigate prevalence and predictors of sustained smoking among male cancer survivors.
\end{abstract}

\section{Materials and Methods}

The Korean National Health Insurance Service-National Health Screening Cohort database was used for this population-based, retrospective study. Study subjects were 15,141 men who were diagnosed with their first incident cancer between 2004 and 2011. Changes in smoking status before and after a cancer diagnosis were investigated. For patients who were current smokers pre-diagnosis, association between post-diagnosis sustained smoking and demographic, socioeconomic, and clinical variables were examined.

\section{Results}

Of the 4,657 pre-diagnosis smokers, 2,255 (48\%) had quit after cancer diagnosis, while $2,402(51.6 \%)$ continued to smoke. In a multivariate logistic regression analysis, younger age at cancer diagnosis (adjusted odds ratio [aOR], 1.37; 95\% confidence interval [Cl], 1.21 to $1.55 ; p<0.001$ ), low socioeconomic status (aOR, $1.29 ; 95 \% \mathrm{Cl}, 1.15$ to $1.45 ; p \leq 0.001$ ), pre-diagnosis heavy smoking ( $a \mathrm{OR}, 1.24 ; 95 \% \mathrm{Cl}, 1.09$ to $1.41 ; \mathrm{p}=0.001$ ), diagnosis of nonsmoking-related cancer (aOR, 1.67; 95\% Cl, 1.42 to $1.96 ; p<0.001$ ), and high serum glucose level (aOR, 1.23; $95 \% \mathrm{Cl}, 1.03$ to $1.46 ; \mathrm{p}=0.019)$ were associated with sustained smoking after a cancer diagnosis.

\section{Conclusion}

Almost half of the male smokers continue to smoke after a cancer diagnosis. Targeted interventions for smoking cessation should be considered for patients with younger age, low socioeconomic status, heavy smoking history, non-smoking-related cancer, and high blood glucose levels.

\section{Introduction}

Smoking has a significant impact on mortality and morbidity of cancer survivors. It is well known that tobacco is an established cause of cancer and numerous chronic diseases, such as cardiovascular and respiratory diseases [1]. Recent studies have suggested that persistent smoking after cancer diagnosis is associated with increased cancer recurrence, sec-

\author{
Key words \\ Cancer survivors, Male smoker, Smoking cessation, \\ Sustained smoking
}


to those without cancer [8-10]. However, up to $70 \%$ of smokers continue to smoke even after a diagnosis of cancer [10-12]. Considering the clinical significance and patients' motivation, the time of cancer diagnosis could be an appropriate time for interventions focusing on smoking cessation [10]. Identifying possible predictive values of sustained smoking might help in targeting the high-risk group when providing this intervention.

Factors considered to affect post-diagnosis smoking in prior reports, albeit inconsistently reported, include age, sex, cancer site, amount of smoking, and level of income [11-20]. However, most of these reports were based on limited sample sizes and specific types of cancer [12-17]. Only a few studies have examined the representative, population-based sample; however, for these studies, data were collected using only one questionnaire after diagnosis and lacked information on longitudinal changes in smoking status, making it difficult to identify differences between quitters and persistent smokers [17-20]. Additionally, data on the Asian population in regard to a diagnosis of cancer and sustained smoking are lacking [21]. Therefore, the aim of this study was to identify changes in smoking behavior after cancer diagnosis and predictors of sustained smoking by examining a large, population-based database from the Korean National Health Insurance (NHIS).

\section{Materials and Methods}

\section{Study population}

This population-based, retrospective study used the Korean National Health Insurance Service-National Health Screening Cohort (NHIS-HealS) data, which is comprised of 514,866 subjects randomly selected from the population that received the national health examination in 2002 and 2003 (10\% of the total). In Korea, NHIS is a mandatory social insurance which covers the entire population in Korea. The subscribers aged over 40 years are provided with a biennial national health screening program, which includes body measurements, laboratory tests, and questionnaires on health behavior. The NHIS-HealS data is comprised of demographic and clinical information on participants extracted from the NHIS database, such as age, sex, insurance premium, residential area, comorbidities, hospital visits, and diagnostic codes, as well as the results of biennial health checkups. The NHIS-HealS participants were followed until 31 December 2013.

Of the individuals included in the database, we limited study subjects to the male cancer patients aged less than 80 years, who were diagnosed with a first incident cancer bet- ween 1 January 2004 and 31 December 2011. We did not include female patients because of the extremely low smoking prevalence (about 6\%) among Korean women [22]. Diagnosis of the first incident cancer was defined as visiting a doctor with a cancer diagnosis code according to the International Classification of Disease, 10th revision (ICD-10) for the first time. To ensure accuracy, patients were included as cancer patients if they: visited the outpatient clinic at least three times with a cancer code; were admitted to the hospital for at least 2 days with a cancer code; received treatment for cancer according to Diagnosis-Related Group (DRG) code within one year of diagnosis. Patients were excluded if they died within 1 year of cancer diagnosis. Of the remaining 24,193 participants, patients who had complete information on pre- and post-diagnosis smoking status (within 5 years prior to and after diagnosis) were included as the final study population (Fig. 1).

\section{Smoking status and changes in smoking}

Data on smoking were obtained from the self-reported questionnaires conducted as a part of the health examinations. The survey items on smoking included smoking status (never smoked/had smoked in the past but quit/smoking currently) and amount of smoking. People who reported that they were currently smoking on their health examination before cancer diagnosis were defined as pre-diagnosis current smokers. The amount of smoking was categorized as heavy ( $\geq 20$ cigarettes/day), moderate (10-19 cigarettes/day), or light $(<10$ cigarettes/day) [23]. Among the pre-diagnosis smokers, people who reported that they were currently smoking on the health check after cancer diagnosis were categorized as sustained smokers, and those who reported not smoking post-diagnosis were categorized as quitters. People who had never smoked pre-diagnosis but started smoking or people who were ex-smokers pre-diagnosis and relapsed after diagnosis were not considered for the analysis on predictors of smoking due to the low prevalence of patients within this group (Fig. 1).

\section{Predictors of smoking}

Sociodemographic, clinical, and lifestyle characteristics of the study population were investigated based on the NHIS claims database and the results of pre-diagnosis health examinations. Body measurements (weight, height, and blood pressure), laboratory tests (blood glucose and cholesterol level), and surveys on lifestyle were conducted at the health checks.

Sociodemographic factors included age at cancer diagnosis $(<50,50-64$, or $65-80$ years), time between cancer diagnosis and post-diagnosis smoking status assessment $(<1,1-2$, or 


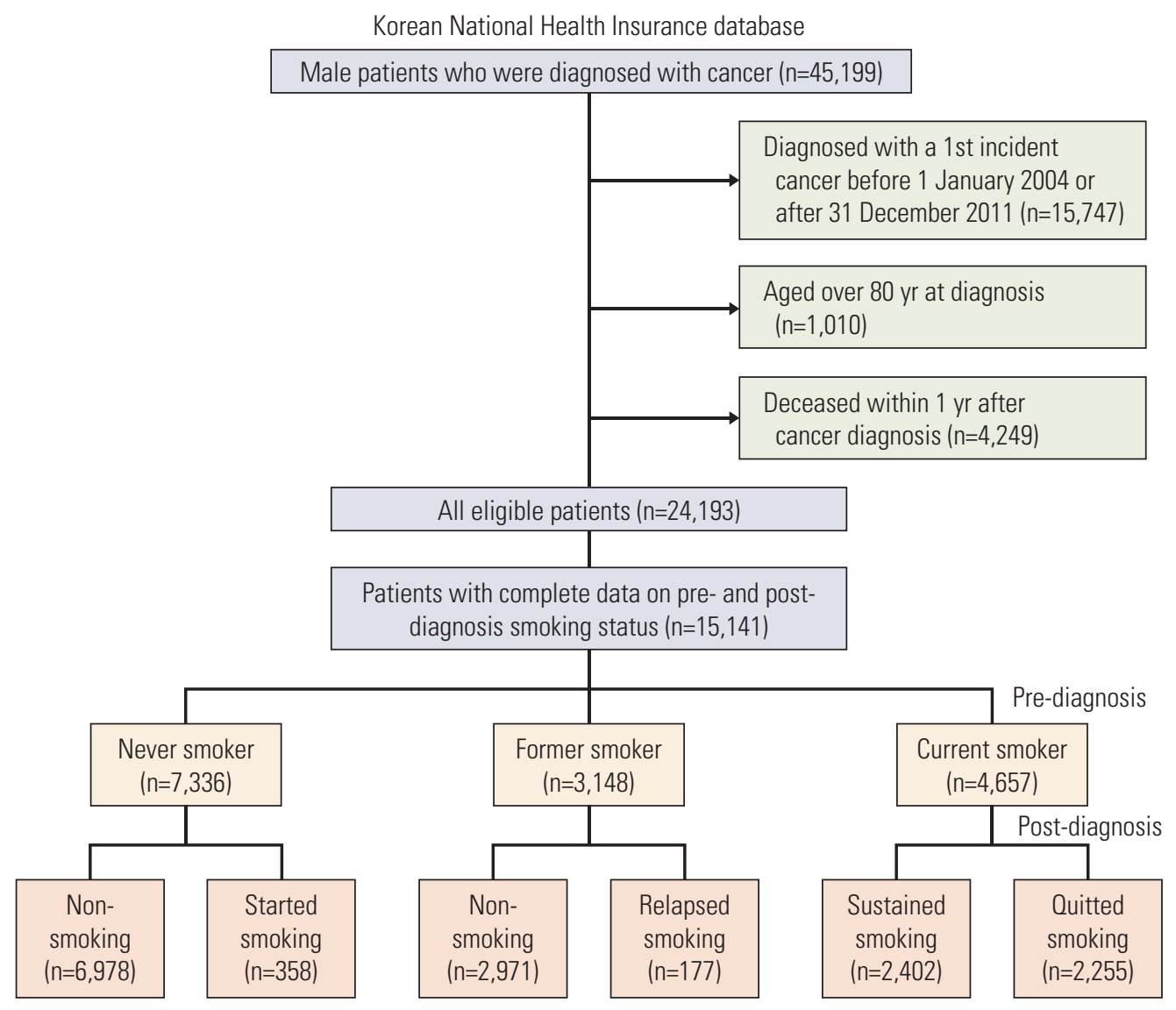

Fig. 1. Summary of patient recruitment and distribution of patients according to smoking behavior.

2-5 years), body mass index ( $<18.5,18.5-25$, or $\left.\geq 25 \mathrm{~kg} / \mathrm{m}^{2}\right)$, socioeconomic status (quartile, based on the level of insurance premium), and place of residence (urban area or country). The lifestyle factors, including amount of smoking (light, moderate, or heavy), physical activity level (none, 1-2, or $\geq 3$ times/wk), and alcohol consumption level (none, 1-2, or $\geq 3$ times/wk) were collected on the self-reported questionnaires at health checks. Clinical factors included type of cancer (smoking-related or non-smoking-related), cancer severity (cancers with low survival or high survival rate), Charlson comorbidity index (CCI) $(0,1,2$, or $\geq 3)$, fasting serum glucose level $(<100,100-125$, or $\geq 126 \mathrm{mg} / \mathrm{dL})$, fasting serum cholesterol level $(<200,200-239$, or $\geq 240 \mathrm{mg} / \mathrm{dL})$, and blood pressure (systolic $<140$ and diastolic $<90$, systolic $\geq 140$ or diastolic $\geq 90$, systolic $\geq 160$ or diastolic $\geq 100 \mathrm{~mm} \mathrm{Hg}$ ). Cancers of the lung, oral cavity, pharynx, esophagus, and larynx were grouped as smoking-related cancers; all other cancers were grouped as non-smoking-related cancers [24]. Cancers with 5-year survival rates under $30 \%$ (cancers of esophagus, liver, gallbladder, pancreas, and lung) were grouped as low survival cancers using the relative survival rates of male patients (1998-2015) described in the Korea Central Cancer Registry data [25]; all other cancers were grouped as high survival cancers. The CCI was calculated based on the diagnosis codes for major comorbidities except for cancer itself [26].

\section{Statistical analysis}

Descriptive statistics were used to determine the pre-diagnosis smoking status and changes in smoking after cancer diagnosis of the study population. Distribution of the cancer sites were described for the pre-diagnosis current smokers. Characteristics of the post-diagnosis sustained smokers and quitters were compared using chi-square or Fisher exact tests. Odds ratios (OR) and 95\% confidence intervals (CI) were estimated by simple logistic regression analysis to evaluate the associations between each variable and sustained smoking. Multivariate logistic regression analysis was then conducted using variables which showed the p-value of $<0.05$ in the univariate analysis. Adjusted odds ratios (aOR) were calculated by adjusting for all related characteristics as 
Table 1. Distribution of cancer sites among patients who were current smokers pre-diagnosis

\begin{tabular}{|lc|}
\hline Types of cancer & No. $(\%)(\mathbf{n}=4,657)$ \\
\hline Smoking-related cancer & $743(16.0)$ \\
\hline Oral cavity and pharynx & $163(3.5)$ \\
\hline Esophagus & $47(1.0)$ \\
\hline Larynx & $65(1.4)$ \\
\hline Lung & $468(10.0)$ \\
\hline Non-smoking-related cancer & $3,914(84.0)$ \\
\hline Stomach & $854(18.3)$ \\
\hline Colon and rectum & $631(13.5)$ \\
\hline Liver & $619(13.3)$ \\
\hline Gallbladder & $65(1.4)$ \\
\hline Skin & $41(0.9)$ \\
\hline Pancreas & $118(2.5)$ \\
\hline Prostate & $752(16.1)$ \\
\hline Kidney & $77(1.7)$ \\
\hline Bladder & $171(3.7)$ \\
\hline Thyroid & $118(2.5)$ \\
\hline Others & $468(10.0)$ \\
\hline
\end{tabular}

determined from the univariate analysis. Separate subgroup analyses were performed in patients aged under 65 years and over 65 years and for each cancer type group (smokingrelated and non-smoking-related).

All analyses were performed using STATA MP statistical software, ver. 14.0 (College Station, TX); statistical significance was defined as two-sided $\mathrm{p}$-values of $<0.05$.

\section{Ethical statement}

This study was approved by the Institutional Review Board (IRB) of the Seoul National University Bundang Hospital (No. X-1701-378-902). The requirement for informed consent was waived by the IRB because of the retrospective nature of the study.

\section{Results}

In total, 15,141 male cancer survivors were included in the study: 4,657 (30.8\%) were current smokers at the pre-diagnosis health checks, 7,336 (48.5\%) had never smoked, and 3,148 $(20.8 \%)$ were former smokers (Fig. 1).

The distribution of cancer sites among the pre-diagnosis current smokers are presented in Table 1 . Overall, $84 \%$ of the patients had non-smoking-related cancers; stomach cancer was the most common type of cancer $(18.3 \%)$, followed by cancers of prostate $(16.1 \%)$, colon and rectum (13.6\%), liver (13.3\%), and lung (10.0\%).

Post-diagnosis changes in smoking status for the study population are summarized in Fig. 1. During a mean followup time of 2.7 years, 2,255 ( $48.4 \%$ ) of the pre-diagnosis current smokers quit smoking, while 2,402 $(51.6 \%)$ reported sustained smoking. Of the sustained smokers, only 278 men $(11.6 \%)$ had reduced their amount of smoking (from heavy to moderate or light, or from moderate to light), and the remaining 2,124 men (88.4\%) maintained the pre-diagnosis smoking amounts (data not shown).

Table 2 shows the association between possible predictor variables and sustained smoking after diagnosis. Of the sociodemographic characteristics, age at cancer diagnosis $(\mathrm{p}<0.001)$ and socioeconomic status $(\mathrm{p}<0.001)$ were associated with sustained smoking in univariate analysis. Of the lifestyle characteristics, pre-diagnosis smoking amount was inversely related to sustained smoking $(\mathrm{p}<0.001)$. Clinical characteristics associated with sustained smoking included type of cancer $(p<0.001)$ and fasting serum glucose level $(\mathrm{p}=0.004)$.

The results of multivariate logistic regression analysis for characteristics associated with sustained smoking are reported in Table 3. Patients were more likely to continue smoking post-diagnosis if they: were younger than 65 years at diagnosis (aOR, 1.37; $\mathrm{p}<0.001$ ); had a low socioeconomic status (less than 50 percentile) (aOR, 1.29; $\mathrm{p}<0.001)$; smoked $\geq 20$ cigarettes per day pre-diagnosis $(\mathrm{aOR}, 1.24 ; \mathrm{p}=0.001)$; had a diagnosis of non-smoking-related cancer $(\mathrm{aOR}, 1.67$; $\mathrm{p}<$ 0.001 ); had a fasting serum glucose level of $\geq 126 \mathrm{mg} / \mathrm{dL}$ (aOR, 1.23; $\mathrm{p}=0.019$ ).

Results from subgroup analyses for each age and cancer type group are presented in Tables 4 and 5. Results of younger patients and patients with non-smoking-related cancer were similar to those found in overall group analysis. Whereas, only non-smoking-related cancer (aOR, 1.94; 95\% CI, 1.48 to $2.54 ; \mathrm{p}<0.001$ ) was associated with sustained smoking in older patients, and only younger age (aOR, 1.67; $95 \%$ CI, 1.22 to $2.28 ; \mathrm{p}=0.001$ ) was associated with smoking in patients with smoking-related cancer.

\section{Discussion}

In this population-based study of male cancer survivors, $52 \%$ of the pre-diagnosis smokers continued smoking after an average 2 years since cancer diagnosis. These results are lower than those reported from previous cohort studies conducted in Western countries $[10,16]$. In a prospective study of the United States, the 2-year smoking cessation rate was 
Table 2. Comparisons of characteristics of the pre-diagnosis current smokers according to post-diagnosis smoking status and univariate analysis for predictors of sustained smoking after cancer diagnosis

\begin{tabular}{|c|c|c|c|c|}
\hline \multirow[b]{2}{*}{ Characteristic } & \multicolumn{2}{|c|}{ Post-diagnosis smoking status, n (\%) } & \multirow[b]{2}{*}{ p-value } & \multirow[b]{2}{*}{$\begin{array}{l}\text { OR }(95 \% \text { CI }) \\
\quad(n=4,657)\end{array}$} \\
\hline & $\begin{array}{c}\text { Sustained } \\
\left.\text { smokers }(n=2,402)^{a}\right)\end{array}$ & $\begin{array}{c}\text { Quitters } \\
\left.(n=2,255)^{\mathrm{a}}\right)\end{array}$ & & \\
\hline \multicolumn{5}{|l|}{ Sociodemographic } \\
\hline \multicolumn{5}{|l|}{ Age at cancer diagnosis (yr) } \\
\hline$<50$ & $408(17.0)$ & $306(13.6)$ & $<0.001$ & $1.57(1.31-1.88)$ \\
\hline $50-64$ & $1,312(54.6)$ & $1,144(50.7)$ & & $1.35(1.19-1.54)$ \\
\hline $65-80$ & $682(28.4)$ & $805(35.7)$ & & 1 \\
\hline \multicolumn{5}{|c|}{ Time since diagnosis to second health check (yr) } \\
\hline$<1$ & $1,051(43.8)$ & $924(41.0)$ & 0.143 & $1.15(0.99-1.32)$ \\
\hline $1-2$ & $747(31.1)$ & 719 (31.9) & & $1.05(0.90-1.22)$ \\
\hline$>2$ & $603(25.1)$ & $608(27.0)$ & & 1 \\
\hline \multicolumn{5}{|l|}{ Body mass index $\left(\mathrm{kg} / \mathrm{m}^{2}\right)$} \\
\hline$<18.5$ & $108(4.5)$ & $94(4.2)$ & 0.824 & $1.07(0.79-1.44)$ \\
\hline $18.5-24.9$ & $1,582(65.9)$ & $1,499(66.5)$ & & $0.98(0.86-1.11)$ \\
\hline$\geq 25$ & $712(29.6)$ & $662(29.4)$ & & 1 \\
\hline \multicolumn{5}{|l|}{ Socioeconomic status, quartile } \\
\hline First, lowest & $695(28.9)$ & $582(25.8)$ & $<0.001$ & $1.44(1.21-1.71)$ \\
\hline Second & $679(28.3)$ & $592(26.3)$ & & $1.38(1.16-1.64)$ \\
\hline Third & $642(26.7)$ & $616(27.3)$ & & $1.26(1.05-1.49)$ \\
\hline Fourth, highest & $386(16.1)$ & $465(20.6)$ & & 1 \\
\hline \multicolumn{5}{|l|}{ Place of residence } \\
\hline Urban area & $1,397(58.2)$ & $1,270(56.3)$ & 0.205 & $1.08(0.96-1.21)$ \\
\hline Country & $1,005(41.8)$ & $985(43.7)$ & & 1 \\
\hline \multicolumn{5}{|l|}{ Lifestyle } \\
\hline \multicolumn{5}{|l|}{ Amount of smoking (pre-diagnosis) } \\
\hline Heavy ( $\geq 20$ cigarettes per day) & $779(32.4)$ & $607(26.9)$ & $<0.001$ & $1.72(1.47-2.03)$ \\
\hline Moderate (10-19 cigarettes per day) & $1,184(49.3)$ & $1,058(46.9)$ & & $1.50(1.30-1.75)$ \\
\hline Light (1-9 cigarettes per day) & 439 (18.3) & $590(26.2)$ & & 1 \\
\hline \multicolumn{5}{|l|}{ Physical activity/wk } \\
\hline None & $1,141(47.6)$ & $1,045(46.5)$ & 0.406 & $1.09(0.95-1.24)$ \\
\hline $1-2$ & $540(22.5)$ & $492(21.9)$ & & $1.09(0.93-1.28)$ \\
\hline 3 or more & $714(29.8)$ & $711(31.6)$ & & 1 \\
\hline \multicolumn{5}{|l|}{ Alcohol consumption/wk } \\
\hline None & $917(38.3)$ & 898 (39.9) & 0.312 & $0.90(0.78-1.03)$ \\
\hline $1-2$ & $718(30.0)$ & $682(30.3)$ & & $0.93(0.80-1.07)$ \\
\hline 3 or more & $759(31.7)$ & $668(29.7)$ & & 1 \\
\hline \multicolumn{5}{|l|}{ Clinical } \\
\hline \multicolumn{5}{|l|}{ Cancer type } \\
\hline Non-smoking-related & $2,098(87.3)$ & $1,816(80.5)$ & $<0.001$ & $1.67(1.42-1.96)$ \\
\hline Smoking-related ${ }^{\mathrm{b})}$ & $304(12.7)$ & 439 (19.5) & & 1 \\
\hline \multicolumn{5}{|l|}{ Cancer severity } \\
\hline Low survival & $689(28.7)$ & $628(27.8)$ & 0.527 & $1.04(0.92-1.18)$ \\
\hline High survival & $1,713(71.3)$ & $1,627(72.2)$ & & 1 \\
\hline \multicolumn{5}{|l|}{ Charlson comorbidity index } \\
\hline$\geq 3$ & $199(8.3)$ & $176(7.8)$ & 0.479 & $1.03(0.83-1.28)$ \\
\hline 2 & $300(12.5)$ & $286(12.7)$ & & $0.95(0.79-1.14)$ \\
\hline 1 & $722(30.1)$ & $722(32.0)$ & & $0.91(0.79-1.04)$ \\
\hline 0 & $1,181(49.2)$ & $1,071(47.5)$ & & 1 \\
\hline
\end{tabular}

(Continued to the next page) 
Table 2. Continued

\begin{tabular}{|c|c|c|c|c|}
\hline \multirow[b]{2}{*}{ Characteristic } & \multicolumn{2}{|c|}{ Post-diagnosis smoking status, n (\%) } & \multirow[b]{2}{*}{ p-value } & \multirow{2}{*}{$\begin{array}{c}\text { OR }(95 \% \text { CI }) \\
(n=4,657)\end{array}$} \\
\hline & $\begin{array}{c}\text { Sustained } \\
\text { smokers }(n=2,402)^{a)}\end{array}$ & $\begin{array}{l}\text { Quitters } \\
(\mathrm{n}=2,255)^{\mathrm{a})}\end{array}$ & & \\
\hline \multicolumn{5}{|l|}{ Fasting serum glucose level (mg/dL) } \\
\hline$\geq 126$ & $350(14.6)$ & $268(11.9)$ & 0.004 & $1.32(1.11-1.58)$ \\
\hline $100-125$ & $719(30.0)$ & $640(28.4)$ & & $1.14(1.00-1.30)$ \\
\hline$<100$ & $1,331(55.5)$ & $1,347(59.7)$ & & 1 \\
\hline \multicolumn{5}{|c|}{ Fasting serum cholesterol level (mg/dL) } \\
\hline$\geq 240$ & $271(11.3)$ & $226(10.0)$ & 0.231 & $1.12(0.92-1.35)$ \\
\hline $200-239$ & $704(29.3)$ & $700(31.1)$ & & $0.94(0.82-1.07)$ \\
\hline$<200$ & $1,424(59.4)$ & $1,327(58.9)$ & & 1 \\
\hline \multicolumn{5}{|l|}{ Blood pressure (mm Hg) } \\
\hline Systolic $<140$ and diastolic $<90$ & $1,746(72.7)$ & $1,621(71.9)$ & 0.828 & $1.04(0.84-1.29)$ \\
\hline Systolic $\geq 140$ or diastolic $\geq 90$ & $463(19.3)$ & 447 (19.8) & & $1.00(0.79-1.23)$ \\
\hline Systolic $\geq 160$ or diastolic $\geq 100$ & $193(8.0)$ & $187(8.3)$ & & 1 \\
\hline
\end{tabular}

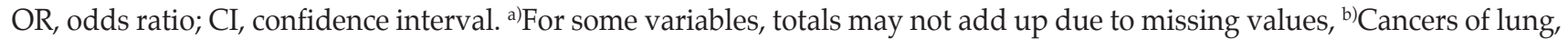
oral cavity and pharynx, esophagus, and larynx were categorized as smoking-related cancer.

Table 3. Multivariate analysis for predictors of sustained smoking after cancer diagnosis in pre-diagnosis current smokers

\begin{tabular}{|c|c|c|c|}
\hline Characteristic & $\mathrm{aOR}^{\mathrm{a})}$ & $95 \% \mathrm{CI}$ & p-value \\
\hline Age $<65 \mathrm{yr}$ at cancer diagnosis (ref. age $\geq 65 \mathrm{yr}$ ) & 1.37 & $1.21-1.55$ & $<0.001$ \\
\hline Socioeconomic status $<50 \%$ (ref. $\geq 50 \%$ ) & 1.29 & $1.15-1.45$ & $<0.001$ \\
\hline Heavy smoker (ref. non-heavy smoker ) & 1.24 & $1.09-1.41$ & 0.001 \\
\hline Non-smoking-related cancer (ref. smoking-related cancer ) & 1.67 & $1.42-1.96$ & $<0.001$ \\
\hline Fasting serum glucose level $\geq 126 \mathrm{mg} / \mathrm{dL}$ (ref. $<126 \mathrm{mg} / \mathrm{dL}$ ) & 1.23 & $1.03-1.46$ & 0.019 \\
\hline
\end{tabular}

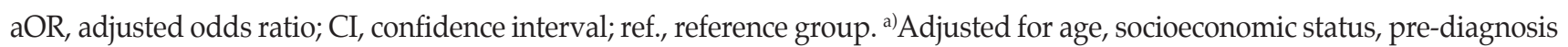
smoking amount, cancer type, and fasting serum glucose level (except for the variable itself).

31\% among 772 smokers diagnosed with cancer [10]. Similarly, in a study of Canadians, 120 of the 175 cancer patients (69\%) continued smoking during an 18-month observation period. Of the 55 patients who quit smoking, 49\% showed a relapse at least once during the study [16]. A possible explanation for this difference is the different distribution of cancer types and severity. In previous studies, patients were limited to those with non-metastatic cancer and patients deemed incurable were typically excluded. Cancer severity defined according to 5-year survival rates was not related to sustained smoking in our study, but further information on individual's cancer stage and prognosis might be necessary to clarify the association between cancer severity and smoking behavior. Also, cancers were mainly limited to non-smoking-related cancers such as prostate, breast, or gastrointestinal tract cancers in previous studies. Smoking-relatedness of cancers might have affected the motivation of patients for smoking cessation, which is in line with our finding that cancer types were related to sustained smoking. Alternatively, our study investigated only male patients. Several previous reports reported that female sex is associated with persistent smoking $[15,27]$.

To our knowledge, only one study has examined the longitudinal changes in smoking behavior after cancer diagnosis in Korea before the present study [21]. The study was based on the 2009 nationwide survey on experiences of cancer survivors executed by the Korean Cancer Center. Information on smoking status at the time of diagnosis and at the time of survey were collected using self-reported questionnaires. Among 439 smokers recruited form 10 cancer centers, reported prevalence of sustained smoking $(27 \%)$ was much lower compared to that in our study. As for the predictors of sustained smoking, alcohol consumption, which was not related to smoking in our study, showed association with continued 
Table 4. Multivariate analysis for predictors of sustained smoking after cancer diagnosis segregated by age at diagnosis

\begin{tabular}{|c|c|c|c|c|c|c|}
\hline \multirow{2}{*}{ Characteristic } & \multicolumn{3}{|c|}{ Age < 65 yr $(n=3,169)$} & \multicolumn{3}{|c|}{ Age $\geq 65$ yr $(n=1,486)$} \\
\hline & $\mathrm{aOR}$ & $95 \% \mathrm{CI}$ & p-value & aOR & $95 \% \mathrm{CI}$ & p-value \\
\hline Socioeconomic status $<50 \%$ (ref. $\geq 50 \%$ ) & 1.31 & 1.14-1.51 & $<0.001$ & 1.24 & $1.00-1.54$ & 0.051 \\
\hline Heavy smoker (ref. non-heavy smoker ) & 1.26 & $1.08-1.46$ & 0.003 & 1.17 & $0.91-1.51$ & 0.217 \\
\hline Non-smoking-related cancer (ref. smoking-related) & 1.53 & $1.25-1.87$ & $<0.001$ & 1.94 & $1.48-2.54$ & $<0.001$ \\
\hline Fasting serum glucose $\geq 126 \mathrm{mg} / \mathrm{dL}$ (ref. $<126 \mathrm{mg} / \mathrm{dL}$ ) & 1.20 & 0.98-1.47 & 0.084 & 1.28 & 0.94-1.79 & 0.120 \\
\hline
\end{tabular}

Adjusted for socioeconomic status, pre-diagnosis smoking amount, cancer type, and fasting serum glucose level. aOR, adjusted odds ratio; CI, confidence interval; ref., reference group.

Table 5. Multivariate analysis for predictors of sustained smoking after cancer diagnosis segregated by cancer type

\begin{tabular}{|c|c|c|c|c|c|c|}
\hline \multirow{2}{*}{ Characteristic } & \multicolumn{3}{|c|}{ Non-smoking-related cancer $(n=3,912)$} & \multicolumn{3}{|c|}{ Smoking-related cancer $(n=743)$} \\
\hline & aOR & $95 \% \mathrm{CI}$ & p-value & aOR & $95 \% \mathrm{CI}$ & p-value \\
\hline Age $<65 \mathrm{yr}$ at diagnosis (ref. age $\geq 65 \mathrm{yr}$ ) & 1.32 & $1.15-1.52$ & $<0.001$ & 1.67 & $1.22-2.28$ & 0.001 \\
\hline Socioeconomic status $<50 \%$ (ref. $\geq 50 \%$ ) & 1.35 & $1.19-1.53$ & $<0.001$ & 1.01 & $0.74-1.37$ & 0.967 \\
\hline Heavy smoker (ref. non-heavy smoker ) & 1.22 & $1.06-1.41$ & 0.005 & 1.30 & $0.94-1.80$ & 0.111 \\
\hline Fasting serum glucose $\geq 126 \mathrm{mg} / \mathrm{dL}$ (ref. $<126 \mathrm{mg} / \mathrm{dL}$ ) & 1.33 & $1.10-1.61$ & 0.004 & 0.83 & $0.54-1.28$ & 0.399 \\
\hline
\end{tabular}

Adjusted for age at cancer diagnosis, socioeconomic status, pre-diagnosis smoking amount, and fasting serum glucose level. aOR, adjusted odds ratio; CI, confidence interval; ref., reference group.

smoking in a previous study. The reasons for discrepancy are unclear, but the specificity of patients visiting national or regional cancer centers might have influenced the differences in observations. Our study investigated the populationbased samples from various clinics and hospitals, which reinforces representativeness.

Overall prevalence of smoking after diagnosis among cancer survivors was $19.4 \%$ in our study, which is about half of the smoking prevalence of general male population in Korea [22]. This outcome is consistent with results of previous investigations on the health behaviors of cancer survivors [8-10]. Data from the U.S. Health and Retirement Study [9] showed that smokers with a new cancer diagnosis were 4.3 times more likely to quit smoking than those without new a diagnosis. Diagnoses of other chronic diseases, such as stroke, heart disease, or lung disease, were also associated with quitting. In a study by Westmaas et al. [10], smoking cessation rates of individuals with cancer diagnosis during 2-year intervals were about 1.6 times higher than healthy controls. These findings imply that the cancer diagnosis could serve as an opportunity for changes in smoking habits. Individuals diagnosed with cancer would be motivated to improve their disease prognosis and more likely to comply with their physicians' advice on health behaviors. Therefore, identifying predictors of persistent smoking and providing the individ- ualized intervention for smoking cessation might have much greater impact among cancer survivors than in healthy adults.

In our study, patients with younger age and patients with lower socioeconomic status were more likely to continue smoking, which is in line with previous reports $[8,11,15,17]$. Younger patients might be in a better general health condition than older patients, which could impede motivations for quitting. Patients with low income might have difficulty in getting additional aid for smoking cessation, including medications, because of the limited financial resource. Pre-diagnosis smoking amount was also related to sustained smoking as in the several previous studies [12,14], which could be explained by the level of nicotine dependence.

Diagnosis of non-smoking-related cancer was strongly associated with persistent smoking. Numerous reports have shown that smoking rates among cancer survivors differ according to types of cancer, although the results may be inconsistent $[8,11,16-19,21]$. In a large population-based study of Australia, lung cancer survivors were 4 times more likely to quit after diagnosis compared with survivors of other cancer types [11]. Previous study in Korea has also reported that lung cancer diagnosis was associated with lower odds of continued smoking (OR, 0.41; 95\% CI, 0.19 to 0.88 ) [21]. A possible reason could be the patients' perceived risk of smoking; A study of cancer patients entering a smok- 
ing cessation program ( $n=111)$ observed that quit motivation was associated with risk perceptions and perceived benefits of quitting [14]. Patients diagnosed with cancers that are strongly linked to tobacco might have been more concerned about smoking and had strong motivation for quitting, while patients with other types of cancers relatively lacked motivation [28].

Baseline high blood glucose level, which was rarely evaluated in the previous investigations, was associated with higher odds of continued smoking in our study. Considering that glucose level is largely affected by dietary intake, dietary habits could be the underlying link. Recent population studies and meta-analysis have demonstrated that smokers tend to have higher blood glucose level and unhealthy nutritional habits compared to non-smokers [29,30]. In studies among cancer survivors, unhealthy habits, such as drinking and smoking, were interrelated [8,31]. Smokers with high glucose level might have unhealthier diets and lifestyles, which could possibly reflect the tendency to stick with their pre-diagnosis smoking habit. Since hyperglycemia and smoking are the established risk factors of cardiovascular disease, smoking cessation might have greater clinical significance in patients with high blood glucose [32]. Our finding that hyperglycemic patients, who are high-risk group of cardiovascular diseases, tend to continue to smoke implies the need for focused interventions for smoking in this population.

When segregated by age and cancer type, socioeconomic status, amount of smoking, and fasting serum glucose level were not associated with sustained smoking in older age group and in smoking-related cancer group. These findings suggest that among patients who already have a high perceived risk of smoking and poor prognosis like older age group and smoking-related cancer group, factors other than age or cancer type might have little impact on smoking behavior.

Recent investigators have focused on the importance of health habit interventions in cancer survivors [33]. While the interventions for smoking, including counseling, cognitive behavioral therapy, and pharmacotherapy, have shown benefits, more systematic, integrated inventions are needed for the routine care of cancer patients [18]. Targeting the highrisk groups found in our study could help effectively providing these interventions. Previous studies have commonly reported that early intervention after the cancer diagnosis is critical and the effective intervention was experienced as an integral part of the primary cancer treatment [33,34], implying that in-hospital smoking cessation program might be necessary during or early after the cancer treatment. Also, a systematic review of smoking cessation programs for cancer survivors has suggested that paying attention to health risk behaviors of survivors and tailoring intervention content to survivors' readiness to quit are important [35]. Using peers to deliver intervention, regular reinforcement of the importance of quitting, and high-intensity delivery over multiple sessions were also shown to be effective. To promote smoking cessation continuously among hard-to-reach patients, various approaches including tailored self-help materials, telephone counseling, and social support by peer group might need to be taken into consideration [36,37]. Integrative intervention combining rigorous in-hospital treatment and tailored long-term support program could be helpful for cancer survivors who have difficulty in quitting smoking.

Our study has some limitations. First, since study subjects were limited to the patients who received at least two times of national health examinations, the result might not be generalizable to all cancer patients. The participants might be more considered about their health and in a healthier lifestyle than general patients. In addition, patients with poor prognosis might have missed their health checks after cancer diagnosis. Second, smoking status was based on the selfreported questionnaires and was not verified via biochemical tests, which might have led to under-reporting of current smoking. Third, while we investigated the numerous possible predictive values of smoking, several factors related to smoking in previous studies, such as second-hand smoking and depression, were not evaluated. Strength of our study is a large sample size and inclusion of various cancer types. Few data exist on longitudinal changes of smoking status in large, population-based samples. Also, we examined the various clinical factors such as blood glucose and cholesterol level.

In conclusion, almost half of the male smokers in Korea continue to smoke after diagnosis of cancer. Patients with younger age, heavy amount of smoking, low socioeconomic status, non-smoking-related type of cancer, and high blood glucose level at baseline are more likely to sustain smoking. Targeted approaches should be considered for these highrisk groups when implementing interventions for smoking cessation.

\section{Conflicts of Interest}

Conflict of interest relevant to this article was not reported.

\section{Acknowledgments}

This study used NHIS-HEALS data (NHIS-2017-2-440) from the National Health Insurance Service (NHIS). The authors received no specific funding for this work. 


\section{References}

1. National Cancer Institute, National Institutes of Health, Department of Health and Human Services. Cancer trends progress report: 2017 update. Bethesda, MD: Natio-nal Cancer Institute; 2017.

2. Do KA, Johnson MM, Lee JJ, Wu XF, Dong Q, Hong WK, et al. Longitudinal study of smoking patterns in relation to the development of smoking-related secondary primary tumors in patients with upper aerodigestive tract malignancies. Cancer. 2004;101:2837-42.

3. Parsons A, Daley A, Begh R, Aveyard P. Influence of smoking cessation after diagnosis of early stage lung cancer on prognosis: systematic review of observational studies with metaanalysis. BMJ. 2010;340:b5569.

4. Sitas F, Weber MF, Egger S, Yap S, Chiew M, O'Connell D. Smoking cessation after cancer. J Clin Oncol. 2014;32:3593-5.

5. Warren GW, Kasza KA, Reid ME, Cummings KM, Marshall JR. Smoking at diagnosis and survival in cancer patients. Int J Cancer. 2013;132:401-10.

6. Duffy SA, Ronis DL, Valenstein M, Fowler KE, Lambert MT, Bishop C, et al. Depressive symptoms, smoking, drinking, and quality of life among head and neck cancer patients. Psychosomatics. 2007;48:142-8.

7. Daniel M, Keefe FJ, Lyna P, Peterson B, Garst J, Kelley M, et al. Persistent smoking after a diagnosis of lung cancer is associated with higher reported pain levels. J Pain. 2009;10:323-8.

8. Park B, Kong SY, Kim J, Kim Y, Park IH, Jung SY, et al. Health behaviors of cancer survivors in nationwide cross-sectional survey in Korea: higher alcohol drinking, lower smoking, and physical inactivity pattern in survivors with higher household income. Medicine (Baltimore). 2015;94:e1214.

9. Keenan PS. Smoking and weight change after new health diagnoses in older adults. Arch Intern Med. 2009;169:237-42.

10. Westmaas JL, Newton CC, Stevens VL, Flanders WD, Gapstur $\mathrm{SM}$, Jacobs EJ. Does a recent cancer diagnosis predict smoking cessation? An analysis from a large prospective US cohort. J Clin Oncol. 2015;33:1647-52.

11. Bryant J, Boyes AW, Hall A, Girgis A, D'Este C, Sitas F. Prevalence and factors related to smoking and smoking cessation 6 months following a cancer diagnosis: a population-based study. J Cancer Surviv. 2016;10:645-53.

12. Bell RJ, Lijovic M, Fradkin P, Schwarz M, Davis SR. Changes in patterns of use of cigarettes and alcohol in women after a first diagnosis of invasive breast cancer: a cohort study of women from Victoria, Australia. Support Care Cancer. 2012; 20:783-9.

13. Eng L, Su J, Qiu X, Palepu PR, Hon H, Fadhel E, et al. Secondhand smoke as a predictor of smoking cessation among lung cancer survivors. J Clin Oncol. 2014;32:564-70.

14. Schnoll RA, Rothman RL, Newman H, Lerman C, Miller SM, Movsas B, et al. Characteristics of cancer patients entering a smoking cessation program and correlates of quit motivation: implications for the development of tobacco control programs for cancer patients. Psychooncology. 2004;13:346-58.

15. Schnoll RA, Martinez E, Langer C, Miyamoto C, Leone F. Pre- dictors of smoking cessation among cancer patients enrolled in a smoking cessation program. Acta Oncol. 2011;50:678-84.

16. Guimond AJ, Croteau VA, Savard MH, Bernard P, Ivers H, Savard J. Predictors of smoking cessation and relapse in cancer patients and effect on psychological variables: an 18-month observational study. Ann Behav Med. 2017;51:117-27.

17. Mayer DK, Carlson J. Smoking patterns in cancer survivors. Nicotine Tob Res. 2011;13:34-40.

18. Eakin EG, Youlden DR, Baade PD, Lawler SP, Reeves MM, Heyworth JS, et al. Health behaviors of cancer survivors: data from an Australian population-based survey. Cancer Causes Control. 2007;18:881-94.

19. Swoboda CM, Walker DM, Huerta TR. Likelihood of smoking among cancer survivors: an updated health information national trends survey analysis. Nicotine Tob Res. 2019;21: 1636-43.

20. Gallaway MS, Glover-Kudon R, Momin B, Puckett M, Lunsford NB, Ragan KR, et al. Smoking cessation attitudes and practices among cancer survivors, United States, 2015. J Cancer Surviv. 2019;13:66-74.

21. Yang HK, Shin DW, Park JH, Kim SY, Eom CS, Kam S, et al. The association between perceived social support and continued smoking in cancer survivors. Jpn J Clin Oncol. 2013;43:4554.

22. Ministry of Health and Welfare, Korea Centers for Disease Control and Prevention. Korea Health Statistics 2015: Korea National Health and Nutrition Examination Survey (KNHANES VI). Cheongju: Korea Centers for Disease Control and Prevention; 2015.

23. Neumann T, Rasmussen M, Heitmann BL, Tonnesen H. Gold standard program for heavy smokers in a real-life setting. Int J Environ Res Public Health. 2013;10:4186-99.

24. U.S. Department of Health and Human Services. Reducing the health consequences of smoking: 25 years of progress: a report of the surgeon general. DHHS Publication No. (CDC) 89-8411. Rockville, MD: U.S. Department of Health and Human Services, Public Health Service, Centers for Disease Control, Center for Chronic Disease Prevention and Health Promotion, Office on Smoking and Health; 1989.

25. National Cancer Center. Annual report of cancer statistics in Korea in 2015. Sejong: Ministry of Health and Welfare; 2017.

26. Sundararajan V, Henderson T, Perry C, Muggivan A, Quan H, Ghali WA. New ICD-10 version of the Charlson comorbidity index predicted in-hospital mortality. J Clin Epidemiol. 2004; 57:1288-94.

27. Burke L, Miller LA, Saad A, Abraham J. Smoking behaviors among cancer survivors: an observational clinical study. J Oncol Pract. 2009;5:6-9.

28. Martinez U, Brandon TH, Sutton SK, Simmons VN. Associations between the smoking-relatedness of a cancer type, cessation attitudes and beliefs, and future abstinence among recent quitters. Psychooncology. 2018;27:2104-10.

29. Tonstad S. Cigarette smoking, smoking cessation, and diabetes. Diabetes Res Clin Pract. 2009;85:4-13. 
30. Alkerwi A, Baydarlioglu B, Sauvageot N, Stranges S, Lemmens $\mathrm{P}$, Shivappa N, et al. Smoking status is inversely associated with overall diet quality: findings from the ORISCAVLUX study. Clin Nutr. 2017;36:1275-82.

31. Vander Ark W, DiNardo LJ, Oliver DS. Factors affecting smoking cessation in patients with head and neck cancer. Laryngoscope. 1997;107:888-92.

32. Coutinho M, Gerstein HC, Wang Y, Yusuf S. The relationship between glucose and incident cardiovascular events: a metaregression analysis of published data from 20 studies of 95,783 individuals followed for 12.4 years. Diabetes Care. 1999;22: 233-40.

33. Demark-Wahnefried W, Pinto BM, Gritz ER. Promoting health and physical function among cancer survivors: potential for prevention and questions that remain. J Clin Oncol. 2006;24: 5125-31.
34. Lauridsen SV, Thomsen T, Kaldan G, Lydom LN, Tonnesen $\mathrm{H}$. Smoking and alcohol cessation intervention in relation to radical cystectomy: a qualitative study of cancer patients experiences. BMC Cancer. 2017;17:793.

35. de Moor JS, Elder K, Emmons KM. Smoking prevention and cessation interventions for cancer survivors. Semin Oncol Nurs. 2008;24:180-92.

36. Emmons KM, Puleo E, Park E, Gritz ER, Butterfield RM, Weeks JC, et al. Peer-delivered smoking counseling for childhood cancer survivors increases rate of cessation: the partnership for health study. J Clin Oncol. 2005;23:6516-23.

37. Abdelrahim A, Balmer C, Jones J, Mehanna H, Dunn J. Considerations for a head and neck smoking cessation support programme: a qualitative study of the challenges in quitting smoking after treatment for head and neck cancer. Eur J Oncol Nurs. 2018;35:54-61. 